\title{
EMDR as an Integrative Therapeutic Approach for the Treatment of Separation Anxiety Disorder
}

\author{
Michelle Morrissey \\ Spanish Peaks Behavioral Health Centers, Pueblo, Colorado
}

\begin{abstract}
This case study reports the use of eye movement desensitization and reprocessing (EMDR) and family therapy for a 10-year-old boy with severe separation anxiety disorder (SAD). It illustrates how the use of the standard EMDR protocol for the boy and his mother combined with family therapy, led to symptom alleviation and restored appropriate developmental functioning as evidenced by behavioral outcomes. The participant initially presented with severe anxiety about separating from his mother, several years after his parents went through a painful divorce. Treatment focused on processing the boy's disturbing memories of past nontraumatic events in 14 EMDR sessions; his mother received 4 EMDR sessions to address her perceived marital failure and guilt about the effects of her ensuing depression on him. Eight family therapy sessions were used to help the family spend positive time together. Prior to treatment, the child had been unable to play outside, checked on his mother frequently, and could not attend activities without her. At the end of treatment, he was able to play with friends outside, ride his bike around town, engage in after school activities, and sleep over at his friends' houses. Gains were maintained at 6 -month follow-up. Treatment did not include instruction in parenting skills or psychoeducation for the mother, or any exposure therapy for the child.
\end{abstract}

Keywords: EMDR; childhood separation anxiety disorder; adaptive information processing model; integrative family therapy

A ccording to Santucci, Ehrenreich, Trosper, Bennett, and Pincus (2009), separation anxiety disorder (SAD) among children is responsible for almost half of all mental health referrals. Many of the children diagnosed with SAD do not participate in developmentally appropriate activities such as sleepovers, camp, dances, or birthday parties. According to Ceyhan and Ceyhan (2009), children who experience SAD are more likely to have an external locus of control and self-perceptions of inadequacy.

Research that focused on parent-child relationships concluded that the maintenance of anxiety disorders can be attributed to overinvolved or overprotective parents. Parents, who maintain a higher percentage of emotional regulation for their children, have children who are at a higher risk for anxiety disorders (Santucci et al., 2009). Other research has found that children who have anxiety disorders are more prone to be bullied and neglected (Comer et al., 2012).

Children diagnosed with SAD are more at risk for sleeping disorders, substance abuse, and developing anxiety and depressive disorders in adolescence and adulthood (Comer et al., 2012). Lewinsohn, HolmDenoma, Small, Seeley, and Joiner (2008) determined that female adults who had SAD as children were less prone to abuse substances than their male counterparts but more prone to panic disorders and depressive disorders.

\section{Symptoms of Separation Anxiety Disorder}

Children diagnosed with SAD experience severe worrying thoughts. These worries encompass thoughts of future separations, parental injuries or death, and fears their parents will leave them. Children with SAD will go to many lengths to avoid these fears and worries. They may be clingy or cry when separation is imminent. They may have tantrums, refuse to attend school, refuse to attend field trips, refuse after school care, or other social activities (Allen, Lavallee, Herren, Ruhe, \& Schneider, 2010). These children's behaviors can be perceived as oppositional. If these children are 
not treated, approximately $36 \%$ will have symptoms that follow them into adulthood.

\section{Associated Symptoms and Diagnosis}

The following includes some of the diagnostic and associated symptoms of SAD (American Psychiatric Association, 2000):

- Symptoms are clinically significant and impact several domains lasting for at least 4 weeks.

- Recurrent distress regarding separation from attachment figures often needing to know where they are and staying in touch with them.

- Feelings of homesickness when away from home causing physical symptoms such as headaches, stomachaches, nausea, vomiting, dizziness, truancy from school, and so forth.

- Preoccupation with fears of accidents, illnesses, or injuries to attachment figure when apart.

- Clingy behaviors that include following attachment figure around the house.

- Nightmares about catastrophes that might befall the family like fires, burglars, murders, kidnappers, and so forth.

- Symptoms are considered developmentally inappropriate and excessive.

\section{Treatment}

According to Rockhill et al. (2010), cognitive behavioral therapy (CBT) and medication management, specifically selective serotonin reuptake inhibitors (SSRIs), are recommended treatments for moderate to severe separation anxiety. Specific skills in this type of treatment include parenting skills to model and support change, exposure therapy with systematic desensitization to increase tolerance of anxiety provoking stimuli, and challenging the cognitive distortions to decrease stress. Vallance and Garralda (2011) recommended the same type of treatment and added psychoeducation and behavior management to the list of treatment.

\section{Research on CBT Treatment}

Jarrett and Ollendick (2012) used a program that was 8 weeks long and included CBT family-based interventions. However, these results showed shortterm improvement in symptoms consistent with CBT treatment. Silva, Gallagher, and Minami (2006) studied various anxiety disorders in children that used CBT treatment. They found that even though CBT produced significant results in several of the anxiety disorders, homework and client motivation altered individual results and they were unsure which CBT interventions were responsible for the most change. Rockhill et al. (2010) reported that anxiety remission rates when using CBT alone was $39 \%$.

\section{Eye Movement Desensitization and Reprocessing}

Eye movement desensitization and reprocessing (EMDR) targets disturbing memories or images, the negative cognitions (NCs) associated with those memories or images, and the corresponding emotions and locations. Positive cognitions are what clients would like to believe about themselves now. The validity of these positive cognitions are then rated on a 7-point scale with 1 being completely false and 7 being completely true. Clients are asked to notice the most disturbing part of the memory with the NC, emotion, and location while bilateral stimulation (BLS) is implemented. BLS is accomplished through the use of eye movements in which clients are instructed to follow their therapist's fingers or lights on a light bar. BLS can also be achieved through alternately tapping on the clients' hands or with the use of theratappers.

\section{EMDR's Theoretical Model}

The theoretical model underlying the application of EMDR is Shapiro's (2001) adaptive information processing (AIP) model. It basically states that how our brain stores memories forms the basis for human perceptions, attitudes, and behaviors. Human experiences are translated by our perceptions into stored memories. Early trauma can disrupt the normal functioning of the AIP system, thereby storing implicit memories that contain disturbing sensations, emotions, and perspectives of the trauma. Similar experiences can then be linked to this dysfunctionally stored material triggering old sensations or emotions.

During EMDR, it is assumed that the AIP system helps to shift the dysfunctionally stored implicit episodic memories to explicit semantic memories. Wherein, clients gain an adaptive resolution allowing them to make new connections to the old experiences. These new connections then become integrated into the memory system with a new positive meaning and are deemed to be functionally stored and available for future use.

\section{EMDR Treatment of Children}

In the treatment of children, many authors recommend the integration of EMDR with CBT and/ or family therapy (Tofani, 2007). This integrated approach of using EMDR and family systems therapy is thought to accelerate changes in the family system 
that can alter behavioral patterns, restore functioning, and improve resources and outcomes (Tofani, 2007). According to Shapiro, Kaslow, \& Maxfield (2007), sometimes family therapy is needed so that family members can reprocess their behaviors or feelings that may be perceived as distressing to the client. This may allow family members to be a better source of support for the client.

In her formulation of EMDR, Shapiro (2001) distinguishes between what she calls "large-T traumas" and "small-t traumas." Large-T traumas are the lifethreatening types of incidents that meet diagnostic criteria as a traumatic event; small-t traumas are minor incidents that are emotionally disturbing and that may have a major impact on the individual (e.g., bullying, rejection, loss).

It should be noted that the research on child treatment with EMDR has focused on symptoms stemming from large-T traumas. Several research studies have shown that EMDR is efficacious in the treatment of children who developed symptoms of posttraumatic stress following large-T traumatic events (see Adler-Tapia \& Settle, 2009; Fleming, 2012; Rodenburg, Benjamin, de Roos, Meijer, \& Stams, 2009). The types of traumas include abuse, accidents (e.g., Kemp, Drummond, $\&$ McDermott, 2010), disasters (e.g., de Roos et al., 2011), and single incidents (e.g., Hensel, 2009). There is also a case series with four children, in which EMDR was effective in eliminating trauma-related choking phobia (de Roos \& de Jongh, 2008).

However, research on EMDR treatment of children with symptoms arising from small-t traumatic incidents is very limited. One study in a child guidance center (Rubin et al., 2001) compared EMDR to standard care for 39 children with a range of problems. The effects for EMDR were not statistically significant and the authors questioned whether "EMDR produces rapid and dramatic improvements with children whose emotional and behavioral problems are not narrowly connected to a specific trauma" (p. 435). More research is needed to investigate the value of EMDR for small-t type traumas that can be ubiquitous in childhood such as bullying, loss, rejection, and failure.

The literature contains little information regarding the use of EMDR with SAD. We were only able to locate a single case report, which was a conference presentation: Mueller (2004) reported the successful use of EMDR for SAD with a comorbid vomiting phobia.

\section{Case Presentation}

"Kent," a 10-year-old male and the younger of two children, came into counseling after suffering from symptoms of SAD for about 3-4 months. Kent's parents divorced a few years ago. Jane had to move away from her ex-husband and the city to receive family support as a single parent. Kent reported numerous symptoms including having a hard time sleeping; having stomachaches at school; worrying about his mother dying, being in a car accident, or falling off of a cliff; and the need to check on his mother's whereabouts several times throughout the day. Kent did not attend any after school activities, he would not play outside with friends, and he did not go on sleepovers. In 2010, Kent was diagnosed with SAD. Because Kent had severe SAD, he insisted on having his mother in sessions with him. As he made progress, Jane would sit just outside the office door. He would interrupt sessions by calling out to her several times to make sure she was still there. Near the end of therapy, Jane was able to sit in a distant waiting room without causing Kent distress.

\section{Presenting Complaints}

In addition to the sleeping issues and checking on his mother's whereabouts, Kent was hypervigilant and would wake up five to six times a night to check on Jane's whereabouts. Kent believed that his mother would get tired of his behaviors and leave him. Kent took to waiting for his mother outside bathroom doors or any place she went where there was a door or a window. He would wait for her to close her car door before he would close his car door. Jane worked at the school where Kent attended. He would text her or call her a couple of times a day to make sure she did not leave school. Kent would not go to friends' houses to play and he would not go on sleepovers. Two weekends a month, Kent would visit his Dad. He reported that he worried about his mother the whole time and would call her several times during the weekend. If Kent did go outside to talk with friends, he would have to check on his mother about every $10 \mathrm{~min}$. During the summer, when Jane worked afternoons, Kent would have to be dragged out of the car kicking and screaming to go to his grandparents' house for daycare. Jane reported increasing tension between Kent and his sister, and she reported less tolerance for his behaviors.

\section{Client History}

Kent is from a divorced family and is the younger of two children. During the history taking, Jane reported no separations from Kent and no large- $T$ traumas. Kent described two small-t traumas. One was being bullied at school and the other one was 2 months 
prior when Kent's friend laid on the football field, refused to get up, and said he was going to die. Kent did not know what to do to help his friend. According to Jane, Kent's symptoms began shortly after this incident with his friend. Jane said that Kent had tubes put in his ears when he was 2-3 years of age. She also conveyed that the divorce occurred when Kent was around 4 years of age. Jane moved the family to a rural community to be near her parents when Kent was 6-7 years of age.

Kent described feeling "anxious" shortly after his parents divorced when he was about 4 or 5 years old. $\mathrm{He}$ indicated that he worried about the safety of his mother or father depending on who he was staying with at the time. He "felt a need" to check on his mother three to four times during the night. He did not "feel" comfortable leaving home to go to school or to the grocery store. He said he "felt" distracted at school and could not think about his studies until he had checked on his mother.

\section{Case Conceptualization and Treatment Plan}

Based on the AIP model of how trauma can disrupt functioning, the theory of EMDR was explained to Jane and to Kent. Despite EMDR recommendations for beginning with the earliest memories, Kent did not want to start there because he had little recall of having tubes put in his ears and the memory was not as disturbing as other more current incidents. Because this was the case, the treatment plan was to use EMDR to process the following four targeted events: the bullying, his friend saying he was going to die, the divorce and the move, and having tubes put in his ears. Treatment would also focus on the possible connection between Kent's friend not getting off the ground, mom's inability to get off the couch, and Kent's NCs of I'm helpless, I don't know what to do, I have no control, and I should have done something.

Kent expressed his fears that his mother would get tired of his behaviors and leave him, or he would get better and she would not spend as much time with him. Family therapy was discussed as a means of addressing these fears and to develop a routine where they spent time together doing things that were enjoyable.

While discussing the course of treatment, Jane stated that she remembered what it was like when her parents divorced, how she had felt responsible, and felt as though she had failed with her marriage. She mentioned how she was so depressed over her marriage ending that she was not able to get off the couch for months and had thoughts about suicide. It was at this time that EMDR therapy for Jane was suggested.
Jane made it clear that medication was a last resort. Methods employed were the therapist's attempt at alleviating symptoms without the referral for medication management. No medication was prescribed or used during this study.

\section{Course of Treatment}

Kent was seen for 26 sessions over a 6-month period. Of the 26 sessions, 14 sessions used the standard EMDR protocol with Kent, 8 sessions used family therapy, and 4 sessions included the standard EMDR protocol with Jane on her issues surrounding her divorce and her parents' divorce. Sessions were twice a week to enhance the process of building rapport and decrease symptoms because of the severe impact it was having on Kent and his family. After Jane completed her four EMDR sessions, Kent's sessions were decreased to weekly because the two of them had experienced symptom reduction. After the first two targets were cleared, Kent experienced some symptom alleviation and he did not want to continue treatment. It became evident that Kent's fear of getting better had influenced his decision to stop treatment. Family therapy sessions were then used and a plan was developed for them to have a game night, a movie night, or a craft night.

Two follow-up sessions were provided after the end of active therapy to achieve closure. This was consistent with the policy of the nonprofit agency to increase time between sessions to determine client's ability to cope and maintain gains while they transition through changes. Kent himself had undergone 14 EMDR sessions to specifically address his traumas.

\section{Preparation Session}

Based on the AIP model of how trauma can disrupt functioning, the theory of EMDR was explained to Kent and his mother. The treatment plan was developed. A safe place was created and the type of BLS was determined. Kent used the light bar with theratappers.

Kent: Target \#1. The target of the first EMDR session was on Kent's experience in music class when he was teased until he cried and the teacher did nothing. Kent's NCs were as follows: "I'm stupid," "I can't get help," and "I don't belong." When asked for positive cognitions (PCs) Kent stated, "I want to learn to feel calm," "I would know what to do," "I can get help," "I'm smart," and "I belong." Kent rated the validity of cognitions (VOCs) to be 1 and 2. He felt sad, disappointed, and upset. He rated the subjective unit of disturbance (SUD) at 10. The location was in his head and stomach. 
Processing began using eye movements (EMs). Between sets, Kent reported more pain in his stomach knowing his teacher did nothing to stop the bullying. His head hurt when he realized everyone was laughing at him. Cognitive interweave was used asking Kent what would make this better. He said, "If the teacher had done her job." Kent was able to release the pain in his head and stomach knowing this would not have happened if she had done her job. He felt next time he could tell his parents and realized bullies are mean to make themselves feel better. SUD rating decreased to 1. PCs were installed and the VOC rating for each increased to 6 and 7. A recheck of the SUD rating showed it decreased to 0 .

Kent: Target \#2. The second EMDR session was on his parents' divorce and how he felt left out because his Dad had a new girlfriend. NCs were "I don't belong," "I am invisible," "It's my fault," and "I should've done something." PCs were "I belong," "I matter" (VOC = 6), "It wasn't my fault," and "I was the best kid I could be" (VOC = 3-4). He felt sadness in his stomach and the SUD rating was a 3. Kent had memories of being at his grandparents when he was told about the divorce. He did not get to see his dad; he felt faint. He was afraid that his mother would leave and never come back. SUD rating decreased to 2, VOCs "It wasn't my fault" and "I was the best kid I could be" increased to 6 and 7, respectfully. Jane called the next day and stated that Kent had only gotten up three to four times that night to check on her. He did not access the school counselor the next day, and he played outside in the yard when she was home.

Kent: Target \#3. Kent came in 4 days later and stated that he slept through the night and he still had not gone to the school counselor's office. The next session targeted Kent's memory of when his friend told him he was going to die and would not get off the ground. NC was "I can't handle it"; PC was "I can learn to handle it"; $\mathrm{VOC}=2$; emotions were anger and fear; the SUD rating was a 10; and the location was everywhere. Kent was very distressed by the image that his friend would not get up off the ground, laying there like a corpse. He could not look at it safely. It is possible that this image was reminiscent of the image he had of his mother who would not get up off the couch. A technique that altered the appearance of the image had to be used to help Kent tolerate the memory. This included having Kent view the image through the opposite end of binoculars and putting the image in black and white. When that did not work, Kent sat in his mother's lap while the BLS was done after he "returned" from looking at the image for $5 \mathrm{~s}$ (Jim Knipe [2008a, 2008b] taught this technique during a workshop on complex PTSD and dissociation). This technique continued until Kent was able to extend "being there" for $20 \mathrm{~s}$ and could return with little distress. This session was on alleviating distress so Kent could return to the standard protocol, it was an incomplete session.

The next session, Kent stated he was "doing awesome": he only checked on his mother two to three times a night, he did not complain of stomachaches, and he still had not accessed the school counselor. Kent was able to view the image of his friend on the ground, NC was "I'm not safe"; PC was "I'm safe now"; $\mathrm{VOC}=5$; emotions were scared and nervous; the SUD rating was a 5; and the location was an ache in his head. Between sets, Kent realized he was safe and that nothing his friend said was true. He felt everything was going to be okay and he was fine; alleviation of ache in the head. Kent noticed the image was not bothersome anymore and that it moved far away. He noticed some nervousness in his stomach and once that was gone Kent said, "I'm safe now, I've got to say my prayers and trust it in God's hands." Those VOCs were now at 6. PC was "I can only control what's in my power to control"; had a VOC of 7; and the SUD rating had decreased to a 1. Each time Kent reported doing well or that he engaged in more independent behaviors, slow BLS was used to reinforce his successes.

Kent: Target \#4. The last EMDR target was on Kent having tubes put in his ears. The full EMDR protocol was not used because Kent could not remember much about this incident. Considering his age, the memories he had were emotional and stored in his body. This was evident during reprocessing when Kent stated there were bright lights and his throat hurt. He began scratching at his throat stating it hurt and he could not breathe. Kent sat in Jane's lap while the BLS continued. Kent was able to release the fear and the pain. The therapist asked Jane if it had been reported that Kent had woken up during surgery. Jane stated it was not reported to her, but she knew something was wrong at the time and suspicioned that this had happened because she herself had woken up during two surgeries. Kent's almost innate fear of not being able to be away from his mother or something bad would happen was linked to this last target. After this session, Jane reported that Kent had stayed home with his sister for $30 \mathrm{~min}$ while she went to the grocery store. He was also able to sleep through the night for the first time in a long time. 


\section{Jane's EMDR Sessions}

Kent's therapist used EMDR with Jane without Kent present. Jane processed through the depression that came after the realization that her husband had cheated and that he wanted to be out of the marriage. She felt she was emotionally vacant for about a year when Kent was about 4 years of age. She said that she did not get up off the couch, she had just lain there, and her daughter would make sandwiches and care for Kent. Jane reported that Kent would "check in on her frequently" during that time. Jane reprocessed the depression and guilt along with her thoughts of responsibility regarding Kent's current situation. Jane also reprocessed her parents' divorce and her responsibility for her brother at a young age.

\section{Family Therapy Sessions}

Kent's underlying fear was that he would get better and his mother would not spend as much time with him. Kent and Jane had to develop new ways of interacting with each other. Family therapy sessions consisted of developing family interactions that were focused on positive interactions versus symptom management. Kent's suggestions were game time, hobby time, and/ or movie night. This time was prescribed to occur once to twice a week for 30-60 $\mathrm{min}$.

\section{Treatment Outcome}

Six weeks after EMDR concluded, Kent was able to go on a sleepover, he was riding his bicycle around town with friends, and he had joined an afterschool program. Jane reported that Kent was sleeping through the night and she could use the restroom without Kent waiting for her at the door. She stated that Kent's relationship with his sister improved because they were no longer fighting but actually spending time playing together. Kent no longer felt the need to check on his mother's whereabouts throughout the day.

Six months after treatment, Kent had maintained all gains. He traveled with the science program out of town without contacting his mother and without Jane attending. Kent had made friends at school and he played outside like any other 11-year-old boy. Jane was grateful for her son being able to live a regular life and she was grateful to have her freedom back.

\section{Implications and Recommendations}

This case study did not attend to modeling parenting skills. It did not attend to psychoeducation of childhood anxiety disorders. There was neither imposition of systematic desensitization on the child nor were there assumptions that the mother's parenting style was overprotective. This case study was conceptualized solely on the basis of Kent's distress about the divorce and his mother's ensuing depression, which was complicated by Jane's perceived marital failure and ensuing emotional detachment. The treatment plan was based around this and an integrated treatment approach was used. This treatment approach included the use of the standard EMDR protocol on Kent, his mother, and family therapy.

This case study illustrates how the standard EMDR protocol helped to alleviate the symptoms and behaviors that accompanied Kent's SAD. The SAD was best understood by the AIP's model of how dysfunctionally stored material could be triggered by a similar event, thought, or feeling, thus bringing about Kent's symptoms. In Kent's case, this occurred when Kent's friend had lain on the ground, refused to get up, and said that he was going to die. This event triggered the earlier memory Kent had of his mother laying on the couch, not getting up because she was so depressed over the end of her marriage, and Kent's fear that she was going to die. Kent's traumatic history included his parents' divorce, his mother's emotional abandonment as she tried to cope with the end of her marriage, and his waking up during surgery. All of these incidences were stored as implicit memories with disturbing sensations and emotions, and all of it occurred prior to Kent's sixth birthday. EMDR was appropriate in reprocessing Kent's past events, allowing him to engage in age-appropriate activities.

Based on the AIP model, future implications regarding the use of EMDR with SAD or an EMDR integrated approach toward treatment should be considered to alleviate symptoms related to previous traumas and real or perceived abandonment with the client and within the client's identified family. This therapist has treated at least three other children with SAD. However, those children all had large-T traumas of house fires that resulted in the death of siblings or parents. Through an integrated approach of family therapy and EMDR, the children's symptoms were alleviated. All of the children began engaging in ageappropriate activities with gains maintained 6 months after treatment.

In Kent's case, it was the first time that this therapist initiated EMDR with a parent. This was caused in part by both parties having had dysfunctionally stored material that was being triggered by current events. Jane's past, in which her parents divorced and she was responsible for her little brother, was activated by her current divorce and her daughter being responsible for Kent. As long as Jane was depressed from both 
occurrences and parenting out of guilt, Kent would only allow himself to progress so far. While Kent was symptomatic, his mother was off the couch and engaged in alleviating his symptoms. Their interactions needed to change for Kent to get better. The concept of Kent holding onto his symptoms to help his mother was posed to Jane as the reason she needed her own EMDR sessions.

\section{Discussion}

The therapist chose to have the mother present to help alleviate Kent's fears and anxiety, to provide emotional support, and to facilitate empathy. In most of these cases, the therapist assesses the parental / guardian bond to determine if their presence will be beneficial. When it is deemed beneficial, the parents / guardians are asked to stay. Several of the SAD cases that this therapist has worked on were children older than the age of 9 years and all involved some level of trauma. If no trauma existed, it is unclear if EMDR would be an appropriate treatment for developmental SAD.

CBT interventions were not considered because this case was not considered developmental SAD. Mom was a single mother who felt overburdened with her new responsibilities of being a provider. She felt guilty over her assumed responsibility for her son's current diminished level of functioning. To add interventions that required more effort for her or homework for them to practice would have been too much. This therapist feared that added expectations would cause this client and his family to drop out of therapy. Because of the severity of Kent's symptoms, the main goal was to reestablish age-appropriate functioning. Because Kent's symptoms were small-t trauma based, EMDR was chosen to resolve the traumas.

EMDR has been viewed as a large-T trauma treatment. However, this case study highlights the devastating effects of multiple small-t traumas as was evidenced by Kent's behaviors and symptoms prior to EMDR treatment. In therapy, sometimes small-t traumas can be overshadowed by large- $T$ traumas. It is important for therapists to perform a complete history taking that includes potential small-t traumas.

Jane's EMDR therapy allowed her to let go of responsibility and guilt surrounding her son, her parents' divorce, and her divorce. Jane's burden was evidenced by her hunched shoulders, somewhat flat affect, and emotional distance from Kent despite physical proximity. After treatment, Jane was more lighthearted in her interactions with Kent. She was smiling, made jokes, and was more playful with him. Jane stated that the prescription to spend time together lasted only a couple of months. She said they naturally found the time to spend together as a family and Kent ended up spending a lot of time with friends and at afterschool activities. Six months after treatment, Jane had changed jobs, lost weight, began dating, and enrolled in college.

It is this therapist's opinion that had EMDR not been used with Jane, her emotional reactivity to past traumas would have loomed over the family potentially interfering with Kent's progress and the family dynamics. Had family therapy not been incorporated into treatment, Kent would not have allowed himself to get better because he was afraid his mom would spend less time with him. The combination of all three treatments made the difference in this family's overall functioning versus making a difference in Kent's overall functioning.

\section{Limitations and Suggestions}

Limitations of this case study included no pretreatment or posttreatment outcome measurements. The only outcome measurements were behavioral. These results cannot be generalized to a larger population. Recommendations for future studies should include the use of standardized measurements for pretreatment and posttreatment. This would lend credence to research studies and offer evidenced-based treatment options to current standards of practice. Future studies should also include evaluation of therapist's fidelity in treatment application. Suggestions for future research include data collection methods that would allow for a greater understanding of SAD and its etiologies. Future research could investigate researcher hypotheses about patterns and commonalities among those with SAD. The assertions and commonalities could lead to different treatment approaches (Creswell, 2012) or standards of practice for this disorder. Future research could also compare an EMDR/family therapy approach with CBT applications in a randomized clinical trial. Other studies could treat children from diverse cultural and ethnic backgrounds and different socioeconomic statuses to allow for the generalization of treatment outcomes across a broader spectrum.

\section{References}

Adler-Tapia, R., \& Settle, C. (2009). Evidence of the efficacy of EMDR with children and adolescents in individual psychotherapy: A review of the research published in peer-reviewed journals. Journal of EMDR Practice and Research, 3(4), 232-247.

Allen, J. L., Lavallee, K. L., Herren, C., Ruhe, K., \& Schneider, S. (2010). DSM-IV criteria for childhood separation anxiety disorder: Informant, age, and sex differences. Journal of Anxiety Disorders, 24(8), 946-952. 
American Psychiatric Association. (2000). Diagnostic and statistical manual of mental disorders (4th ed.). Washington, DC: Author.

Ceyhan, A., \& Ceyhan, E. (2009). Relationship between early separation anxiety and depressive symptoms: The mediating role of locus of control and problem solving skills. Pakistan Journal of Social and Clinical Psychology, 7(1), 3-17.

Comer, J. S., Puliafico, A. C., Aschenbrand, S. G., McKnight, K., Robin, J. A., Goldfine, M. E., \& Albano, A. M. (2012). A pilot feasibility evaluation of the CALM Program for anxiety disorders in early childhood. Journal of Anxiety Disorders, 26(1), 40-49.

Creswell, J. W. (2012). Qualitative inquiry and research design: Choosing among five approaches (3rd ed.). Thousand Oaks, CA: Sage.

de Roos, C., \& de Jongh, A. (2008). EMDR treatment of children and adolescents with a choking phobia. Journal of EMDR Practice and Research, 2(3), 201-211.

de Roos, C., Greenwald, R., den Hollander-Gijsman, M., Noorthoorn, E., van Buuren, S., \& de Jongh, A. (2011). A randomised comparison of cognitive behavioural therapy (CBT) and eye movement desensitisation and reprocessing (EMDR) in disaster-exposed children. European Journal of Psychotraumatology, 2(5694). http:// dx.doi.org/10.3402/ ejpt.v2i0.5694

Fleming, J. (2012). The effectiveness of eye movement desensitization and reprocessing in the treatment of traumatized children and youth. Journal of EMDR Practice and Research, 6(1), 16-26. http://dx.doi. org/10.1891/1933-3196.6.1.16

Forgash, C., \& Knipe, J. (2008a). Integrating EMDR and ego state treatment for clients with trauma disorders. In C. Forgash \& M. Copeley (Eds.), Healing the heart of trauma and dissociation with EMDR and ego state therapy. New York, NY: Springer Publishing.

Hensel, T. (2009). EMDR with children and adolescents after single-incident trauma an intervention study. Journal of EMDR Practice and Research, 3(1), 2-9.

Jarrett, M., \& Ollendick, T. (2012). Treatment of co-morbid attention-deficit/hyperactivity disorders and anxiety in children: A multiple baseline design analysis. Journal of Consulting and Clinical Psychology, 80(2), 239-244.

Kemp, M., Drummond, P., \& McDermott, B. (2010). A wait-list controlled pilot study of eye movement desensitization and reprocessing (EMDR) for children with post-traumatic stress disorder (PTSD) symptoms from motor vehicle accidents. Clinical Child Psychology and Psychiatry, 15(1), 5-25.

Knipe, J. (2008b). Loving eyes: Procedures to therapeutically reverse dissociative processes while preserving emotional safety. In C. Forgash \& M. Copeley (Eds.), Healing the heart of trauma and dissociation with EMDR and ego state therapy. New York, NY: Springer Publishing.
Lewinsohn, P., Holm-Denoma, J., Small, J., Seeley, J., \& Joiner, T. (2008). Separation anxiety disorder in Childhood as a risk factor for future mental illness. Journal of American Academy of Child and Adolescent Psychiatry, 47(5), 548-555. http://dx.doi.org/10.1097/ CHI.0b013e31816765e7

Mueller, K.-M. (2004). Separation anxiety disorder and vomit phobia: An integrated approach. Paper presented at the 2nd Annual Conference of the EMDR UK \& Ireland Association, Birmingham, United Kingdom.

Rockhill, C., Kodish, I., DiBattisto, C., Macias, M., Varley, C., \& Ryan, S. (2010). Anxiety disorders in children and adolescents. Current Problems in Pediatric and Adolescent Health Care, 40(4), 66-69.

Rodenburg, R., Benjamin, A., de Roos, C., Meijer, A. M., \& Stams, G. J. (2009). Efficacy of EMDR in children: A meta-analysis. Clinical Psychology Review, 29(7), 599-606.

Rubin, A., Bischofshausen, S., Conroy-Moore, K., Dennis, B., Hastie, M., Melnick, L., Reeves, D., \& Smith, T. (2001). The effectiveness of EMDR in a child guidance center. Research on Social Work Practice, 11(4), 435-457.

Santucci, L., Ehrenreich, J., Trosper, S., Bennett, S., \& Pincus, D. (2009). Development and preliminary evaluation of a 1-week summer treatment program for separation anxiety disorder. Cognitive and Behavioral Practice, 16(3), 317-331. http://dx.doi.org/10.1016/j .cbpra.2008.12.005

Shapiro, F. (2001). Eye movement desensitization and reprocessing: Basic principles, protocols, and procedures. New York, NY: Guilford Press.

Shapiro, F., Kaslow, F., \& Maxfield, L. (Eds.). (2007). Handbook of EMDR and family therapy processes. Hoboken, NJ: John Wiley \& Sons.

Silva, R., Gallagher, R., \& Minami, H. (2006). Cognitivebehavioral treatments for anxiety disorders in children and adolescents. Primary Psychiatry, 13(5), 68-76.

Tofani, L. R. (2007). Complex separation, individuation processes, and anxiety disorders in young adulthood. In F. Shapiro, F. W. Kaslow, \& L. Maxfield (Eds.), Handbook of EMDR and family therapy processes (pp. 265-283). Hoboken, NJ: John Wiley \& Sons.

Vallance, A., \& Garralda, E. (2011). Anxiety disorders in children and adolescents. In D. Skuse, H. Bruce, L. Dowdney, \& D. Mrazek (Eds.), Child psychology and psychiatry: Frameworks for practice (2nd ed.). Chichester, United Kingdom:John Wiley \& Sons. http: / / dx.doi.org/ 10.1002 /9781119993971.ch27

Correspondence regarding this article should be directed to Michelle Morrissey, Spanish Peaks Behavioral Health Centers, 1304 Chinook Lane, Pueblo, CO 81001. E-mail: MichelleM@spanishpeaks.org 